\title{
Pressure's on: is it time to move ahead with nonhuman primates?
}

A s the father of a child who succumbed to globoid cell leukodystrophy (Krabbe disease), Dr. Leon Martel was passionate about his research to find a cure for this autosomal recessive neurological disorder, for which there is no satisfactory treatment. Martel's initial gene therapy research at Great Eastern University used mice for modeling the disease, and he found increased longevity, improvement of clinical signs, and no adverse side effects attributed to the therapy. He then progressed to treating affected dogs. Bone marrow transplantation, combined with or without gene replacement therapy, showed similar early indications of success, although some signs of mild liver and neural toxicity were found postmortem in two treated normal control animals.

Martel's work was published and presented at meetings, which eventually led to a phone call from his U.S. senator, who served on the Health, Education, Labor and Pensions Committee. The senator urged Martel and the college dean to push ahead with testing on rhesus monkeys as these nonhuman primates were previously used for Krabbe disease research. The senator's altruistic goal was to have Martel accumulate enough data for the school to apply for accelerated approval of the procedure from the Food and Drug Administration and then begin clinical trials with afflicted human children. Nevertheless, after initial talks between Martel, the dean, and the chair of the IACUC, it became obvious that Martel and the IACUC chair were hesitant to move forward with nonhuman primate studies until more work was done to elucidate the cause of the mild toxicity seen in the dog studies. The dean, under continuing pressure from the senator, argued that the mouse studies showed no toxicity at all and that the mild toxicity in dogs had no overt clinical impact and was found in only two of the twelve control animals. The discussion led to a key question: If affected and nonaffected monkeys were to be studied, what clinical signs would be used to determine if there was either improvement or toxicity to the animals? Clinical signs in affected monkeys were known ${ }^{1}$, but clinical signs in normal monkeys subjected to Martel's gene therapy technique were unknown. Martel feared that given the infrequent and mild aberrant findings in dogs and the long life span of rhesus monkeys, there may no simple way for him to determine a clear and meaningful study endpoint.

If you were Martel, how would you deal with the pressure from the dean? Would you submit a protocol amendment to add monkeys to the study? Is there any federal regulation or policy that prohibits the senator from pressuring the dean, Martel, or the IACUC?

\section{Jerald Silverman}

University of Massachusetts Medical School,

Worcester, MA, USA.

e-mail:Jerald.Silverman@umassmed.edu

Published online: 19 September 2019

https://doi.org/10.1038/s41684-019-0393-8

References

1. Baskin, G. B. et al. Lab Anim Sci. 48, 476-482 (1998).

\section{Animal welfare is the primary concern}

T his scenario illustrates one of the more difficult situations encountered by an IACUC: the balance between animal welfare and scientific progress. Martel developed a hopeful treatment for a rare but very serious disorder that affected his own child. After a promising study in mice he moved on to testing his treatment in dogs. Although successful, two of the control animals showed signs of mild liver and neural toxicity on necropsy. Martel was contacted by his U.S. Senator, who urged him to move to a rhesus monkey model so that the treatment could progress to human trials ${ }^{1,2}$.

Martel and the IACUC chair were hesitant because of the issues found on the dog necropsies and a concern over whether they would be able to identify a good study endpoint in a long-lived animal, such as a macaque. The senator urged Great Eastern's dean to encourage the nonhuman primate (NHP) project and the dean is now pressuring Martel and the IACUC chair to move forward.

We agree with the PI and the IACUC chair in this case that caution is indicated. The liver and neural toxicity in canine subjects is concerning because it was found in treated members of the control group, not affected animals. It warrants further testing to isolate the cause before moving to the (NHP) model. The Animal Welfare Act $(\$ 2143, \mathrm{~b}, 1)$ charges the IACUC with representing "society's concerns regarding the welfare of animal subjects used" at the institution. Animal welfare must be the primary concern of any IACUC. Further, PHS Policy IV.C.8 states: "Applications and proposals that have been approved by the IACUC may be subject to further appropriate review and approval by officials of the institution. However, those officials may not approve an activity involving the care and use of animals if it has not been approved by the IACUC." The authority to approve this work resides only with the IACUC. While both the dean and the senator may have the best intentions, their attempts to pressure the PI and the IACUC chair to begin work that neither is comfortable with should not be allowed. Martel and the IACUC chair should enlist support from the campus veterinarian and the Institutional Officer if needed to ensure the IACUC is able to do its job without any outside pressure.

\section{Maria Reaves* and Becky Northcut}

Texas State University, San Marcos, TX, USA.

*e-mail:mer226@txstate.edu

Published online: 19 September 2019 https://doi.org/10.1038/s41684-019-0396-5

References

1. United States Department of Agriculture. Animal Welfare Act and Animal Welfare Regulations.

2. Office of Laboratory Animal Welfare, National Institutes of Health. Public Health Service Policy on Humane Care and Use of Laboratory Animals. (US Department of Health and Human Services, Bethesda, Maryland, USA, 2015). 